\title{
ESTILOS DE APRENDIZAJE INTERCULTURALES(SENSIBILIDAD INTER(ULTURAL)Y MODALIDADES EN LA CARTA ARGUMENTATIVA EN ESTUDIANTES BILINGÜES DE LICENCIATURA EN LENGUAS MODERNAS DE UNA UNIVERSIDAD COLOMBIANA Y UNA FRANCESA
}

\author{
INTERCULTURAL LEARNING STYLES(INTERCULTURAL SENSITIVITY) AND \\ MODALITIES IN ARGUMENTATION LETTER IN BILINGUAL STUDENTS IN \\ MODERN LANGUAGES FROM A COLOMBIAN AND A FRENCH UNIVERSITY
}

Neira Loaiza Villalba', PhD.

Universidad del Quindío

\section{Claudia Elizabeth Ortiz Ruiz², Mg.}

Universidad del Valle

\section{RESUMEN}

En el campo de las diferencias individuales y de la formación bilingüe, en particular de futuros profesores de lenguas extranjeras, los estilos de aprendizaje que los estudiantes

$1 \quad P h D$. en Ciencias de la Educación, coordinadora de la línea de investigación en bilingüismo de la Maestría y el Doctorado en Ciencias de la Educación de la Universidad del Quindío. Líder del Grupo de Investigación ESAPIDEX$B$ y profesora de francés del Programa de Licenciatura en Lenguas Extranjeras con Énfasis en Inglés y Francés de la misma universidad. Correo electrónico: nloaiza@uniquindio. edu.co ORCID: 0000-0002-8541-4231.

$2 \quad M g$. en Didáctica del Francés como lengua Extranjera de la Universidad Jean Monnet. Miembro del Grupo de Investigación interinstitucional ESAPIDEX-B (Universidad del Quindío-Universidad del Atlántico), del grupo EILA de la Universidad del Valle y profesora del área de Francés del departamento de Lenguas y Culturas Extranjeras de esta última. Correo electrónico: claudia.elizabeth.ortiz@correounivalle.edu.co ORCID: 0000-0002-1562-6861. despliegan en los procesos de aprendizaje de una segunda lengua son influenciados por la cultura propia en interacción con la cultura asociada a la lengua extranjera, configurando rasgos afectivos, psicológicos y cognitivos que, si bien son individuales, responden también a características culturales ligadas a su comunidad de origen. Son los estilos de aprendizaje interculturales (Leiva, 2015; Jiménez, 2004), concepto que reconoce aspectos socioculturales de los estilos de aprendizaje (Keefe, 1988), que en esta investigación, se centran en la sensibilidad intercultural como dimensión de la interculturalidad, asumida como habilidades sociales que el individuo podría desarrollar en 
contextos de contacto de lenguas y culturas (Chen y Starosta, 2000).

En este sentido, la habilidad argumentativa escrita, tipología textual que predomina en la educación superior, es influenciada por los estilos de aprendizaje asociados a la sensibilidad intercultural, lo cual se refleja en las modalidades del discurso argumentativo (Gosselin, 2010). Es así como, desde las teorías del bilingüismo escolar y las ciencias del lenguaje, este estudio de caso cualitativo describe cómo los estilos de aprendizaje, ligados a la sensibilidad intercultural, se reflejan en la competencia argumentativa escrita bilingüe en términos de las modalidades discursivas en una carta argumentativa en L1 y L2 en estudiantes bilingües de licenciatura en lenguas modernas (francés y español LE) de dos universidades, una colombiana y una francesa. En general, los resultados muestran un perfil de estilo de aprendizaje en donde hay una presencia significativa del estilo reflexivo y del estilo verbal $y$ un equilibrio entre lo sensorial y lo intuitivo, y entre lo secuencial y lo global. En cuanto a la sensibilidad intercultural, se evidencia una actitud favorable y neutra alta que facilita la interacción intercultural. A partir del análisis de retórica contrastiva intra e interindividual, se encuentra que las modalidades sobresalientes son la epistémica, la alética y la deóntica que validan las representaciones desde lo objetivo, lo subjetivo y/o lo institucional.

\section{PALABRAS CLAVE}

$\begin{aligned} & \text { Estilos de aprendizaje interculturales, } \\ & \text { sensibilidad intercultural, } \\ & \text { modalidades, } \\ & \text { argumentación escrita bilingüe. }\end{aligned}$

\section{ABSTRACT}

In the field of individual differences and bilingual training, in particular of future foreign language teachers (FL), the learning styles that students display in the learning processes of a second language are influenced by their own culture in interaction with the culture associated with the FL, configuring affective, psychological and cognitive traits that, although they are individual, they also respond to cultural characteristics linked to their community of origin. The intercultural learning styles (Leiva, 2015; Jiménez, 2004) is a concept that recognizes sociocultural aspects of learning styles (Keefe, 1988), which in this research focus on intercultural sensitivity as a dimension of interculturality, assumed as social skills that the individual could develop in contexts of contact between languages and cultures (Chen and Starosta, 2000).

In this sense, the written argumentative skill, a textual typology that predominates in higher education, is influenced by the learning styles associated with intercultural sensitivity, which is reflected in the modalities of argumentative discourse (Gosselin, 2010). This is how, from the theories of school bilingualism and language sciences, this qualitative case study describes how learning styles, linked to intercultural sensitivity, are reflected in bilingual written argumentative competence in terms of discursive modalities in a argumentative letter in L1 and L2 in bilingual undergraduate students in modern languages (French and Spanish FL) from two universities, one Colombian and one French. In general, the results show a profile of learning styles where there is a significant presence of the reflective style and the verbal style and a balance between the sensory and the intuitive, and between the sequential and the global. Regarding intercultural sensitivity, a high favorable and neutral attitude is evidenced that facilitates intercultural interaction. From the analysis of intra and interindividual contrastive rhetoric, it is found that the outstanding modalities are the epistemic, the alethic and the deontic that validate the representations from the objective, the subjective and / or the institutional perspective. 


\section{KEYWORDS:}

Intercultural Learning styles, intercultural sensitivity, modalities, bilingual written argumentation

\section{INTRODUCCIÓN}

En la trama de la sociedad globalizada del siglo $\mathrm{XXI}$, el bilingüismo constituye un desafío lingüístico, académico y profesional para los ciudadanos, y una responsabilidad que los gobiernos, a través de sus políticas educativas, han puesto en manos del sistema escolar y de los docentes de lenguas segundas o extranjeras. Su importancia se debe a la movilidad laboral, estudiantil y cultural, al turismo, al auge de las tecnologías virtuales, principal ventana al conocimiento y al establecimiento de acuerdos comerciales internacionales (Abdallah-Pretceille, 2006; Grosjean, 2010). En este sentido, las TIC favorecen el contacto de estudiantes de diferentes lenguas y culturas proporcionando ambientes de aprendizaje auténticos y posibilidades de desarrollar o perfeccionar habilidades comunicativas, al tiempo que es posible establecer relaciones interculturales, que permiten un enriquecimiento tanto lingüístico como cultural. Enmarcado en las teorías del bilingüismo escolar y las ciencias del lenguaje, este estudio de caso ${ }^{3}$ describió cómo los estilos de aprendizaje (EA) interculturales, abordados desde el concepto de sensibilidad intercultural, permean la competencia argumentativa escrita bilingüe (francés y español) en términos de las modalidades discursivas de estudiantes de licenciatura en lenguas extranjeras (LE) de dos universidades públicas, colombiana y francesa.

En la formación bilingüe de futuros docentes de LE los rasgos afectivos, psicológicos y cognitivos son influenciados por la cultura propia, las convenciones que el uso colectivo y

3 Este artículo reporta los resultados del proyecto de investigación 953 adscrito a la Vicerrectoría de Investigaciones de la Universidad del Quindío. las instituciones han establecido históricamente a nivel social, educativo, lingüístico y discursivo. Por ello, los EA asociados al contacto entre sujetos de distintas culturas y comunidades de habla, han ido fortaleciéndose como tema de interés de la comunidad investigativa (Leiva, 2015; Jiménez, 2004) en el contexto educativo en países de inmigración, en especial como herramienta conceptual que facilita la promoción de estrategias didácticas facilitando la adaptación de los estudiantes extranjeros al sistema educativo del país de acogida. Para este estudio de caso, no inscrito en un contexto de inmigración, el concepto de EA interculturales se articuló con el concepto de sensibilidad intercultural como rasgo de la dimensión afectiva de la competencia intercultural, en tanto habilidad social que implica una disposición emocional de los sujetos de diversas culturas a comprender y a apreciar las diferencias individuales y colectivas, promoviendo un comportamiento apropiado y efectivo en una situación de interacción intercultural.

Por otra parte, la investigación sobre EA en la formación bilingüe ha evidenciado que éstos influencian no sólo la adquisición de las lenguas, sino que se plasman en el uso de ellas (Loaiza y Galindo, 2014), como se pudo confirmar en las dinámicas escriturales de estudiantes colombianos y franceses examinadas en este estudio de caso. Dado que, en el contexto universitario, la habilidad de escribir textos hace parte de las competencias escriturales que deben alcanzar los estudiantes tanto en su L1 como en su L2 (Loaiza, 2016), se escogió la tipología argumentativa. De este modo, se identificaron, en los textos argumentativos, los mecanismos lingüísticos y discursivos que los participantes utilizan para modalizar su argumentación en L1 y L2 teniendo en cuenta la dimensión de la sensibilidad intercultural en relación con los EA. 
Algunos estudios sobre EA interculturales se han centrado en los contextos de inmigración o multiculturales (Santos-Sopena, 2016; Leiva, 2015; Jiménez, 2004; Park, 1997), relacionándolos con los métodos de enseñanza y las dificultades de aprendizaje de los estudiantes extranjeros 0 de comunidades marginadas, cuyos estilos no corresponden con los desarrollados por el sistema educativo del país, lo que ocasiona en ellos un bajo rendimiento académico. Igualmente se han interesado por las diferencias perceptuales entre estudiantes de diferentes nacionalidades (Park, 1997).

Por otra parte, se encuentran muy pocos estudios sobre los EA interculturales, en términos de la sensibilidad intercultural, y los que se hallan se desarrollan en ámbitos de inmigración (ámbito exolingüe). Algunos de ellos examinan la sensibilidad intercultural en estudiantes de primaria en relación con variables étnicas, de edad y de género, como se observa en el estudio de Sanhueza y Cardona (2009) en España. Los resultados revelan que los niños y niñas poseen una sensibilidad intercultural positiva con una actitud pasiva hacia la interacción. Otro estudio, en contexto de lengua extranjera, también en España, examina la competencia intercultural y la sensibilidad intercultural en una muestra de estudiantes de coreano (LE) identificando las dimensiones de la escala de sensibilidad intercultural de Chen y Starosta (2000), y relacionándolas con variables como el nivel de escolaridad, relaciones con extranjeros, experiencia en el extranjero, número de idiomas que domina y género. Los resultados arrojan que los estudiantes tienen una sensibilidad interculturalidad bastante elevada, en especial en las dimensiones de respeto en la interacción y la capacidad de disfrute en la interacción, una particularidad de los resultados es que el nivel de estudio está directamente relacionado con el desarrollo de la sensibilidad intercultural (Jeon, 2018).
Con relación a estudios sobre modalidades en el texto argumentativo, ha habido pocos desarrollos en el campo de la formación bilingüe que trasciendan una visión "estrecha" del uso de los verbos modales (Gosselin, 2010). Así, Deshors (2014) estudia los patrones de uso de los verbos modales de may y can en la interlengua de estudiantes franceses y chinos de inglés, encontrando que, si bien ambos tipos de población comparten un esquema de construcción modal común para may y can, difieren en la forma en que manifiestan el esquema en términos lingüísticos.

Deigual manera, en Colombia se han desarrollado algunos estudios como los de Cardona et al. (2017) quienes encontraron en estudiantes de licenciatura en lenguas extranjeras, la tendencia a argumentar desde la emotividad y lo experiencial en L1 y L2 evidenciando el uso de modalizadores apreciativos. Asimismo, en el contexto colombiano, la modalización ha sido estudiada en lengua materna por Ramírez (2013) quien se centra en el análisis de noticias, en prensa escrita, sobre la violencia en Colombia. Los resultados muestran que, si bien los hechos son verídicos y relevantes, están cargados de subjetividad, concluyendo que no son posibles las representaciones neutras, o puras, debido a las posturas ideológicas de los periodistas.

Teniendo en cuenta estos vacíos en la investigación sobre los EA interculturales, en términos de la sensibilidad intercultural, y las modalidades, este estudio buscó responder a la siguiente pregunta de investigación: ¿De qué manera los EA interculturales, en términos de la sensibilidad intercultural, se reflejan en la modalización discursiva en un texto argumentativo escrito en su L1 y L2 en una muestra de estudiantes de licenciatura en lenguas extranjeras de dos universidades públicas, una colombiana y una francesa? 
El estudio de caso de corte cualitativo indaga, a través del análisis de retórica contrastiva intra e interindividual, sobre las influencias interlingüísticas e interculturales, ligadas a las modalidades direccionadas por los EA asociados a la sensibilidad intercultural en los textos argumentativos escritos en L1 y L2. Los resultados mostraron la utilización de diferentes mecanismos lingüísticos y discursivos en cuanto a las modalidades, de acuerdo con su perfil de EA, y permeados por las dimensiones de la sensibilidad intercultural, entre ellas: el autocontrol, la apertura de mente, la implicación en la interacción, el respeto por las diferencias culturales y la empatía.

El estudio se sustentó en los siguientes conceptos:

Bilingüismo: Grosjean (1982, 2008), entre otros autores, ve el bilingüismo con un carácter integral en reacción a la visión monolingüe del bilingüismo que considera que el bilingüe debería tener dos competencias lingüísticas separadas y aisladas, es decir dos monolingües en una persona. Para este autor, el bilingüe es un interlocutor completamente competente que en su vida cotidiana usa dos o más lenguas de acuerdo con unas necesidades específicas contextuales, que no necesariamente implica un dominio equivalente de ambas lenguas o de sus habilidades lingüísticas receptivas y productivas, incluso en su L1.

EA interculturales: Para Leiva (2015), los EA se definen como "un conjunto de preferencias, tendencias y disposiciones personales que tiene un sujeto para hacer algo y que se manifiesta a través de un patrón conductual y de distintas fortalezas (actitudinales, emocionales, éticas, procedimentales) que lo hacen distinguirse de los demás" (p. 40). Más recientemente el concepto de EA se ha relacionado con la interculturalidad, de ahí que se hable de los EA interculturales, cuyos desarrollos teóricos son todavía muy incipientes. Leiva reconoce "cómo esos EA están mediatizados y modulados por la configuración de contextos educativos donde la interculturalidad se ha implementado a través de determinados programas, unidades didácticas o acciones específicas" (2015: 36). Dado la amplitud del concepto de interculturalidad en los EA, se toma el concepto de sensibilidad intercultural de Chen y Starosta (2000) como una dimensión afectiva y sociocultural de los EA.

Sensibilidad intercultural: El enfoque intercultural favorece las relaciones entre personas de diversas culturas e identidades haciendo énfasis más en las relaciones que en las diferencias. En este sentido, la comunicación entre estos grupos se concibe como la competencia comunicativa intercultural, comprendiendo habilidades cognitivas y afectivas que facilitan la interacción intercultural en un contexto social y cultural determinado (Vilà, 2003). Para Chen y Starosta $(1996,1997,2000)$ la sensibilidad intercultural, como componente afectivo de la competencia intercultural, es el conjunto de habilidades sociales $y$ comportamentales que favorecen el encuentro intercultural a partir de las dimensiones de autocontrol, apertura de mente, implicación en la interacción, respeto por las diferencias culturales y empatía, entre otras.

Modalidades: Para Gosselin, las modalidades lingüísticas corresponden a los modos de "validación de las representaciones" (2010: 50), expresadas en los enunciados. Esquemáticamente, las modalidades son "aquello que debe ser agregado a las representaciones para que haya la constitución de juicios" (Gosselin, 2000: 53). A dichas modalidades se les expresa por medio de marcadores gramaticales, construcciones sintácticas, o un conjunto de lexemas unidos; además, se les puede hallar en el plano material a nivel del texto en sus diversas expresiones, tanto oral como escrito. Los parámetros 
conceptuales definen las categorías modales: alético, de la realidad objetiva; epistémico, de la realidad subjetiva; deóntico, del deber y el permiso; volitivo, de la voluntad; apreciativo, de lo deseable e indeseable; y axiológico, de lo loable y lo punible por la institución.

\section{Competencia argumentativa escrita bilingüe:}

Como proceso y producto de la acción comunicativa social, el acto argumentativo presenta una secuencia básica constituida por una hipótesis (premisa) y una conclusión, entre las cuales se establece una relación de probabilidad y credibilidad. Para que la conclusión sea creíble debe existir, en términos de Van Dijk (1978), una relación semántica condicional entre las circunstancias (hechos) que soportan dicha conclusión, es decir los argumentos deben sustentarse en una garantía o legitimidad, un refuerzo, una justificación o una explicación. La competencia argumentativa escrita bilingüe se asume como "la capacidad de un sujeto bilingüe para producir, en contextos comunicativos y socioculturales, y con base en un conjunto de conocimientos y habilidades, textos argumentativos escritos, en una o más lenguas adicionales a su LM" (Loaiza, 2016: 110).

\section{METODOLOGÍA}

Se trata de una investigación educativa, cualitativa y descriptiva con diseño de estudio de caso que implementó la metodología de análisis retórico contrastivo en la escritura argumentativa bilingüe, examinando "las diferencias discursivas existentes entre las diversas lenguas y las distintas expectativas que caracterizan a los miembros de una determinada comunidad lingüística..." (Martin, 2000). La muestra estuvo constituida por estudiantes colombianos (español L1 y francés L2) y franceses (francés L1 y español L2) de nivel B1+ en LE de licenciatura en lenguas extranjeras de dos universidades públicas (una colombiana y una francesa) a quienes se les aplicó el inventario de EA (Felder y Soloman, 1998), el CELD argumentativas en L1 y L2, la escala de sensibilidad intercultural de Chen y Starosta (2000) y una tarea de escritura argumentativa en L1 y L2. Para el análisis de las modalidades en los textos se implementó una plantilla analítica.

\section{RESULTADOS Y DISCUSIÓN}

\section{RESULTADOS DEL CUESTIONARIO DE EA ASOCIADOS A LA SENSIBILIDAD INTERCULTURAL Y DEL CUESTIONARIO DE ELD ARGUMENTATIVAS EN L1 Y L2}

\section{Diagnóstico de EA}

En general, los resultados muestran un perfil de $E A$, en el cual hay una presencia significativa del estilo reflexivo y del estilo verbal y un equilibrio entre lo sensorial y lo intuitivo, y entre lo secuencial y lo global. Igualmente, el equilibrio entre estilos sensorial-intuitivo, secuencialglobal —que más que dicotómicos sugieren un continuum - indicaría la flexibilidad cognitiva de los estudiantes, para quienes funcionarían de manera similar tanto canales de percepción de la información concretos, prácticos, orientados a los hechos y procedimientos, como canales conceptuales, mediados por la escritura o la lengua hablada

\section{Diagnóstico de la sensibilidad intercultural asociada al estilo de aprendizaje}

El nivel de sensibilidad intercultural de colombianos y franceses resultó muy cercano, $4.0 / 5$ y $4.1 / 5$, los franceses con el mayor puntaje. Los niveles favorable y neutro alto en los resultados permiten intuir que las dimensiones cognitivas, afectivas y comportamentales (Chen y Starosta, 2000), presentan rasgos relacionados con las dimensiones de la sensibilidad intercultural. Esto sugiere una disposición emocional de los estudiantes a comprender y a apreciar diferencias individuales y grupales, facilitando la interacción intercultural. 
Si asociamos los EA detectados y el nivel de sensibilidad intercultural, observamos que no hay un patrón estable: tanto el participante de mayor puntaje $(4.75)$ como el de menor $(3,8)$, entre los franceses, presentan mayor equilibrio entre estilos bimodales. Entre los colombianos el mayor puntaje presenta mayor equilibrio entre sus estilos bimodales, pudiéndose presumir que la sensibilidad intercultural podría ser favorecida por la flexibilidad que supone el equilibrio entre estilos bimodales.

\section{Diagnóstico de elecciones lingüístico- discursivas argumentativas en L1 y L2}

Para el análisis de resultados del cuestionario de elecciones lingüístico-discursivas (CELD) argumentativas en L1 y L2, se consideró la incidencia de los EA (Felder y Silverman, 1988) y el tipo de argumento asociados a características lingüístico-discursivas de cada cultura, en una situación comunicativa hipotética, mostrando los resultados:

En la primera parte del cuestionario, los colombianos escogieron, en la primera pregunta, argumentos direccionados por el estilo reflexivo tanto en $L 1$ como en $L 2$, patrón que los franceses mantuvieron en L1, pero no en L2. Sin embargo, resalta que los colombianos mayoritariamente optaron en las otras tres preguntas por argumentos afines al estilo activo, mientras que los franceses se inclinaron por argumentos afines al estilo reflexivo. En la segunda parte del cuestionario, relacionada con las elecciones lingüístico-discursivas (ELD) permeadas por la cultura de origen, los estudiantes en general escogieron, en ambas lenguas, argumentos más racionales, puntuales y más formales, que caracterizan culturalmente a la lengua francesa, como lo expresa Fischer (2009). En nuestra cultura, la argumentación se basa más en lo emotivo que en lo reflexivo y es menos formal. En este sentido, se esperaba que los estudiantes colombianos escogieran argumentos marcados por la emotividad e informalidad, talvez porque ya han tenido una formación lingüística en inglés, francés y español han desarrollado una escritura más académica, cercana a las formas escriturales argumentativas francesas.

\section{Perfil de los participantes según los EA asociados a la sensibilidad intercultural y a las ELD argumentativas bilingües}

El análisis de retórica contrastiva intraindividual, es decir, desde la perspectiva de las dos lenguas del sujeto bilingüe, muestra el siguiente perfil de los participantes teniendo en cuenta sus respuestas en los diferentes cuestionarios aplicados.

Participante colombiano 1: Su perfil de EA presenta tendencia moderada en los estilos reflexivo, intuitivo, verbal y global, reflejándose en la escogencia lingüística y discursivamente idéntica en L1 y L2, la cual coincide en los estilos reflexivo y global. Estos resultados se podrían relacionar con la reflexividad presente en los estilos reflexivo, intuitivo y verbal, y podrían permear sus ELD apoyando, según Chen y Starosta (2000), una disposición emocional y cognitiva a comprender diferencias individuales. Además, el estudiante escogió, en L1 y L2, argumentos más cercanos a las formas escriturales francesas y menos a las de su cultura. Los primeros facilitan la interacción intercultural, al reflejar respeto por el otro, autocontrol y empatía (Chen y Starosta, 2000).

Participante colombiano 2: Su perfil de tendencia moderada en los estilos reflexivo y visual, y el equilibrio en los estilos bimodales sensorial-intuitivo y secuencial-global encajan, en L1 y en L2, con las ELD que corresponden a los estilos reflexivo, intuitivo y secuencial, los dos últimos bimodales. Los resultados arrojan una actitud favorable en la sensibilidad intercultural, presentando rasgos cognitivos y 
afectivos relacionados con la racionalidad ligada a los estilos reflexivo, intuitivo y secuencial. En el CELD priman, en L1 y L2, los argumentos asociados a los rasgos escriturales franceses que apoyarían la interacción intercultural.

Participante colombiano 3: Su perfil de EA, de tendencia moderada en los estilos reflexivo, sensorial, verbal y secuencial, coincide, en L1 y L2, con las ELD por ellos direccionadas en su mayoría. En el cuestionario de sensibilidad intercultural, el estudiante obtuvo --como sus coterráneos-- un nivel neutro alto, asociado a rasgos cognitivos, afectivos y comportamentales que promueven el entendimiento intercultural y a la escogencia de argumentos cercanos a las formas escriturales francesas en $L 1$, a diferencia de los argumentos más emotivos y menos formales en L2.

Participante francés 1: En su perfil de EA predomina el estilo activo de tendencia moderada y el equilibrio en las demás categorías bimodales. En el CELD, en L1 y L2, optó por argumentos direccionados por el estilo visual y secuencial, difiriendo, en el estilo reflexivo e intuitivo para L1 y activo y sensorial para L2. En cuanto al cuestionario de sensibilidad intercultural, se mantuvo una actitud neutra alta. La escogencia de argumentos formales y racionales en ambas lenguas sugeriría la transferencia de rasgos escriturales de la L1 a la L2, verificando la hipótesis de interdependencia lingüística de Cummins (1983).

Participante francés 2: Su perfil de EA muestra equilibrio en las categorías bimodales activoreflexivo y secuencial-global, tendencia moderada en el estilo intuitivo y tendencia fuerte en el estilo visual. Esta variabilidad se refleja en las ELD en L1 y L2, las cuales coinciden solamente en el estilo secuencial; sobresaliendo en L1 los estilos reflexivo, sensorial y visual, y en L2 sus opuestos. En el cuestionario de sensibilidad intercultural, mantuvo el patrón general, un nivel neutro alto. En L1 escogió argumentos, tanto formales y racionales propios de su cultura, como emotivos e informales más cercanos a la cultura hispánica, inclinándose por los primeros en L2.

Participante francés 3: Su perfil de EA muestra un equilibrio en la mayoría de las categorías bimodales, excepto en la tendencia moderada del estilo verbal, único estilo en el cual coinciden sus ELD en L1 y L2. En L1, escoge argumentos direccionados por los estilos reflexivo, intuitivo y secuencial, mientras que en L2 la elección es direccionada por los estilos opuestos, lo cual es lógico pues el equilibrio en su perfil le permite transitar entre estilos. Obtiene un nivel favorable en la escala de sensibilidad intercultural y se decide en L1 por argumentos propios de las formas escriturales francesas que transfiere a la L2, facilitando la interacción intercultural.

\section{ANÁLISIS DE RETÓRICA CONTRASTIVA INTRAINDIVIDUAL DE LAS MODALIDADES EN EL TEXTO ARGUMENTATIVO EN L1 Y L2 PERMEADAS POR LOS EA ASOCIADOS A LA SENSIBILIDAD INTERCULTURAL}

El análisis de las modalidades (Gosselin, 2010) partió de identificar, en las cartas argumentativas en L1 y L2, las unidades de sentido y en ellas las predicaciones, en las cuales se identificaron las modalidades. Se retuvieron las sobresalientes, aquellas en las que la fuerza argumentativa aporta más a la validación o invalidación de las representaciones en torno a la tarea de escritura, cuyo tema abordó la actividad de las cam girls.

Participante colombiano 1: De acuerdo con el estilo reflexivo del participante, la modalidad sobresaliente que prima en su texto argumentativo en L1 es la alética, mientras que en L2 la modalidad sobresaliente es la epistémica. Lo anterior sugeriría que en L2 está más presente su voz autoral con una mayor carga emotiva expresando su opinión subjetiva de la realidad, asociado al estilo activo 
que no corresponde a su estilo identificado. La sensibilidad intercultural se refleja en la tarea de escritura, sobresaliendo en L1 la apertura de mente, es decir que el participante invita a la comunidad a trascender visiones tradicionales; por ejemplo: "La visión de la exploración y disfrute de las personas no debería ser vista como un asunto de moralidad...". En L2, "Elles ne devraient pas être jugée comme des personnes qui veulent gagner de l'argent facilement, elles ont seulement trouvé une manière de trouver du plaisir dans leur travail4", expresa empatía, es decir, «pensar los mismos pensamientos y sentir las mismas emociones que la otra persona» (Adler y Towne, 1987, en Chen, 1997).

Participante colombiano 2: En consonancia con el estilo reflexivo del participante, la modalidad sobresaliente en L1 es la alética y, en L2, la epistémica. Presenta una subjetividad enunciativa que remite a la apertura de mente, empatía e implicación en la interacción en L1 y en L2; un ejemplo de esta última: "Así pues, tenemos que replantearnos nuestras bases culturales y críticas para analizar y definir a una persona por lo que realmente es y no por su profesión". El uso de la primera persona del plural es un llamado a los interlocutores a la dignificación de la persona más allá de su condición laboral.

Participante colombiano 3: En relación con el estilo reflexivo del participante, destaca la modalidad sobresaliente alética en L2 $\mathrm{y}$, en L1, la epistémica. La sensibilidad intercultural en los argumentos de la tarea de escritura, en L1, resalta el respeto por las diferencias culturales, el autocontrol, la apertura de mente y la empatía. En algunos argumentos, el autor se muestra favorable a las cam girls que no considera inmorales, pero siendo consciente que entre sus interlocutores puede haber quienes no compartan la visión de que "solo

$4 \quad$ Traducción libre: "Ellas no deben ser juzgadas como personas que quieren ganar dinero fácil, solo que han encontrado una manera de encontrar el placer en su trabajo". se trata de una actividad donde hay intereses particulares", a manera de autocontrol atenúa su discurso, expresando respeto por las diferencias individuales.

Participante francés 1: En relación con el estilo activo, sobresale la modalidad epistémica tanto en L1 como en L2. En la cultura francesa, es frecuente expresar, incluso en los textos académicos, la voz autoral con la primera persona del singular (je) o con la primera del plural (nous/ on), lo cual refuerza la argumentación desde la subjetividad del individuo. Esto se refleja en L1 y L2, donde prima la implicación en la interacción, seguida de la empatía. La primera se aprecia en el ejemplo: "On peut se demander en quoi cette pratique pose-t-elle problème ${ }^{5 "}$ ", en el cual la pregunta indirecta interpela al interlocutor, involucrándolo en la interacción.

Participante francés 2: En relación con el estilo en equilibrio activo-reflexivo del participante, la modalidad sobresaliente en L1 y L2 es la deóntica que expresa la subjetividad desde los juicios regulatorios. La sensibilidad intercultural en L1 y L2 presenta resultados diferentes a los demás participantes, con una visión conservadora de la práctica de las cam girls que la equipara con la prostitución, lo que evidencia poca apertura de mente frente al fenómeno. Asimismo, el diálogo con los interlocutores de la carta es escaso; quizá, por esta razón poco se observa la implicación en la interacción y la empatía. A pesar de su postura, se evidencian rasgos de autocontrol, cuando reconoce la importancia de asegurar el libre ejercicio y la seguridad para quienes realizan esta práctica.

Participante francés 3: En relación con su estilo equilibrado activo-reflexivo, las modalidades sobresalientes en L1 y L2 son la deóntica y la epistémica, respectivamente. Desde las subjetividades, el estudiante modaliza en L1 basado en los juicios regulatorios y en L2 $\overline{5}$ Traducción libre: "Uno puede preguntarse por qué esta práctica es problemática ". 
desde la opinión subjetiva, las representaciones compartidas, la duda. La sensibilidad intercultural se refleja en L1 en la implicación en la interacción, la apertura de mente y el respeto por las diferencias culturales. En el enunciado: "Ainsi, je vous invite à faire en sorte que ces activités intimes n'interviennent pas dans le cadre universitaire et professionnele", la implicación en la interacción se marca en la invitación directa (je vous invite), a los interlocutores. En L2, predomina la implicación en la interacción, seguida de la empatía.

\section{ANÁLISIS DE RETÓRICA CONTRASTIVA INTERINDIVIDUAL DE LOS EA ASOCIADOS A LA SENSIBILIDAD INTERCULTURAL Y A LAS MODALIDADES EN EL TEXTO ARGUMENTATIVO EN L1 Y L2}

El análisis de retórica contrastiva interindividual, es decir, desde la perspectiva de examinar la escritura de las lenguas entre sujetos, señala el siguiente comportamiento de los participantes como grupo:

Los colombianos tendieron a escribir textos más extensos en L1, mientras que los franceses se extendieron más en la L2. Por otro lado, evidencian un empleo similar de las modalidades sobresalientes en L1 y en L2, argumentando los colombianos desde lo alético en L1 y lo epistémico en L2, mientras los franceses desde lo epistémico en L2 y lo deóntico en L1. Como se observa en el enunciado en L1 de un estudiante colombiano: "Ahora bien, si miramos la modalidad 'cam girl' o modelo web cam, es simplemente una profesión como cualquier otra" predomina la modalidad alética, al considerar esta actividad como una realidad objetiva, una profesión. Ahora bien, en el enunciado en L1 de un estudiante francés: "Les autorités devraient concentrer leurs efforts dans la mise en place

$6 \quad$ Traducción libre: "De este modo los invito a que estas actividades íntimas no intervengan en el contexto universitario y profesional". d'une réglementation stricte ${ }^{7 "}$ destaca con el condicional "devraient" la modalidad deóntica, emitiendo un juicio regulatorio.

De manera menos frecuente, las modalidades apreciativas, axiológicas y volitivas estuvieron presentes en ambas lenguas. Por ejemplo, en L2: "La sexualité ne devrait pas être stigmatisée toujours comme des actes immoraux ${ }^{8}$ " (estudiante colombiano) donde se expresa una modalidad apreciativa que emite un juicio de valor sobre la sexualidad. Igualmente, en L1: «En effet, je condamne tout type de chantage ou de harcèlement de la part de ceux qui détiennent des photos ou vidéos compromettantes d'autrui ${ }^{9}$ », el estudiante francés modaliza axiológicamente emitiendo un juicio moral institucional mediante la expresión «je condamne».

\section{CONCLUSIONES}

De manera global, los estudiantes colombianos revelan una tendencia moderada al estilo reflexivo y la distribución de los otros estilos varía en las diferentes categorías bimodales. Para los estudiantes franceses, sus EA tienden a ser mayoritariamente equilibrados entre lo activo y lo reflexivo, lo sensorial y lo intuitivo y entre lo secuencial y lo global. Esta tendencia a lo reflexivo puede sugerir que el proceso de formación bilingüe promueve en los estudiantes un ejercicio de observación, análisis, síntesis, introspección, metacognición que les permite descubrir las regularidades de los dos sistemas lingüísticos, compararlos, contrastarlos, formular hipótesis de funcionamiento de acuerdo con una situación comunicativa, con el contexto social y con la sensibilidad intercultural de cada sujeto.

Por otra parte, para los colombianos las modalidades sobresalientes, alética en L1

\footnotetext{
$7 \quad$ Traducción libre: "Las autoridades deberían concentrar sus esfuerzos en la implementación de una normativa estricta".

8 Traducción libre: "La sexualidad no siempre debería ser estigmatizada como un acto inmoral"

9 Traducción libre: "De hecho, condeno cualquier tipo de chantaje o acoso por parte de quienes tienen fotos o videos comprometedores de otras personas".
} 
y epistémica en L2, sugeriría que en L1 sus argumentos son más descriptivos de la realidad objetiva, mientras que en L2 resalta su argumentación soportada en su subjetividad. $\mathrm{Si}$ bien se esperaría que lo epistémico primara en L1, dado que las culturas latinas se caracterizan por una mayor subjetividad, el hecho de modalizar más desde lo alético, se justificaría porque en su formación como futuros docentes, principalmente en el área de inglés, se ha enfatizado en las características escriturales anglófonas, que han transferido a la escritura en francés con argumentos basados en la información factual, de autoridad, con menos presencia de la voz autoral, rasgos lingüístico-discursivos que son más cercanos a la modalidad alética.

A pesar de que el análisis de retórica contrastiva evidenció algunas características lingüísticodiscursivas comunes a toda la muestra, también es cierto que dejó ver diferencias originadas en los rasgos culturales convencionales de los colombianos y los franceses asumidos como grupos culturalmente diversos.

Para los franceses, las modalidades deóntica en L1 y epistémica en L2, significaría que, en L1, sus argumentos se basan en juicios regulatorios descriptivos y valores instituidos mientras que en L2 sobresale su subjetividad. La recurrencia de la modalidad deóntica podría sugerir una mayor presencia del pensamiento cartesiano característico de esta cultura, su apego y respeto a las normas sociales que, algunos, aun estando en desacuerdo con la práctica de las cam girls, consideren que lo relevante es controlar y proteger a quienes en ella intervienen.

Los resultados de una actitud favorable y neutra alta de los participantes, en la escala de sensibilidad intercultural, obedecería a que su formación como docentes de lenguas y culturas extranjeras, propiciaría un pensamiento más amplio, una mayor comprensión de las diferencias individuales y colectivas. Ello se evidencia en la mayor recurrencia, de las dimensiones de la sensibilidad intercultural, de la implicación en la interacción, la empatía y la apertura de mente en sus argumentos.

Finalmente, los resultados de esta investigación, permitirían comprender que la diversidad en los perfiles de EA, las formas de modalizar el discurso y las dimensiones relacionadas con la sensibilidad intercultural confirman que los individuos son únicos e irrepetibles en su configuración cognitiva, afectiva y comportamental reflejada en sus producciones lingüístico-discursivas argumentativas en L1 y L2.

\section{REFERENCIAS BIBLIOGRÁFICAS}

Abdallah-Pretceille, M. (2006). La communication interculturelle des signes d'appartenance aux symptômes d'une relation. Dans F. Dervin, et E.

Cardona, A., Mery, J., Montoya, A., Muñoz, L., \& Padilla, D. García, J. (dir). (2018). EA y competencia argumentativa escrita bilingüe (español-inglés) desde una perspectiva cultural en estudiantes de $5^{\circ}$ semestre de una Licenciatura en Lenguas Modernas de una universidad pública de Colombia. Informe final proyecto de investigación. Semillero de investigación. ESAPIDEX-B. Universidad del Quindío. Armenia.

Chen, G., \& Starosta, W. (2000). The development and validation of the intercultural sensitivity scale. Human Communication, 3, 2000, 1-15.

Chen, G. (1997). A review of the concept of intercultural sensitivity. ERIC. 
ESTILOS DE APRENDIZAJE INTERCULTURALES (SENSIBILIDAD INTERCULTURAL) Y MODALIDADES EN LA CARTA ARGUMENTATIVA EN ESTUDIANTES BILINGÙES DE LICENCIATURA EN LENGUAS MODERNAS DE UNA UNIVERSIDAD COLOMBIANA Y UNA FRANCESA

Chen, G., \& Starosta, W. (1996). Intercultural communication competence: A synthesis. Annals of the International Communication Association, 19(1), 353383.

Cummins, J. (1983). Interdependencia lingüística y desarrollo educativo de los niños bilingües. Toronto. Universidad de Alberta.

Deshors, S. (2014). Constructing meaning in L2 discourse: The case of modal verbs and sequential dependencies. Glynn, Dylan and M. Sjo. lin (eds.) Subjectivity and Epistenicity: Stance Strategies in Discourse and Narration, 329-348.

Felder, R., \& Silverman, L. (1988). Learning and teaching styles in engineering education. Engineering education, 78(7), 674-681.

Felder R., \& Soloman B. (1998). Index of Learning Styles, Available: [http://www. ncsu.edu/felder-public/lLSpage.html ]

Fischer Hubert, D. (2009). Étude comparative des langues française et espagnole dans le livre bilingue de Paul Dupuy : Abrégé élémentaire des différences les plus remarquables entre la France et l'Espagne (1829). Open Edition Journals, 42, 149-164.

Gosselin, L. (2010). Les modalités en français: la validation des représentations. Brill.

Grosjean, F. (2010). Bilingual. Harvard university press.

Grosjean, F. (2008). Studying bilinguals. Oxford University Press.

Grosjean, F. (1982). Life with two languages: An introduction to bilingualism. Harvard University Press.
Jeon, Y. (2018). Sensibilidad intercultural en la enseñanza de lenguas extranjeras: el caso de la clase de coreano. Tesis inédita de maestría. Universidad Complutense De Madrid, España.

Jiménez, H. (2004). El impacto de las diferencias de los estilos de aprendizaje interculturales en la enseñanza a los alumnos extranjeros de intercambio del etc. Tecnológico de Monterrey, México.

Keefe, J. (1988). Profiling and Utilizing Learning Style. Reston, Virginia, NASSP, p. 48.

Leiva, J. (2007). Educación y conflicto en escuelas interculturales. Spicum. Málaga.

Leiva, J. J. (2015). Interculturalidad y estilos de aprendizaje: nuevas perspectivas pedagógicas. International Journal of Educational Research and Innovation (IJERI), 3, 36-51.

Loaiza, N. \& Galindo, A. (2014). Estilos de aprendizaje de segundas lenguas y formación bilingüe consecutiva en educación primaria, secundaria y superior: hacia un estado del arte. Lenguaje, 42(2), 291-314. Recuperado de http://www.scielo.org.co/scielo. php?script $=$ sci_arttext $\&$ pid $=$ S0120$34792014000200004 \&$ lng=es\&tlng $=$

Loaiza, N. (2016). Estilos de aprendizaje, organización cognitiva y competencia argumentativa escrita bilingüe de sujetos en formación bilingüe de $4^{\circ}$ semestre de licenciatura en lenguas modernas. Tesis inédita de doctorado. Universidad del Quindío.

Martín, P. (2000). La retórica contrastiva: nuevas dimensiones en el análisis del discurso escrito. Revista de Filología de la Universidad de La Laguna,18, 205-217. 
Park, C. (1997). Learning Style Preferences of Korean, Mexican, Armenian-American, and Anglo Students in Secondary Schools. NASSP Bulletin, 81 (585), 103111.

Ramírez L. A. (2013). La representación mediática de la violencia: Modalización polifónica en la noticia del periódico El Tiempo. Discurso \& Sociedad, (4), 719739.

Sanhueza, S. \& Cardona, M. (2009). Evaluación de la sensibilidad intercultural en alumnado de educación primaria Escolarizado en aulas culturalmente diversas. Revista de Investigación Educativa, 27 (1), 247-262.

Santos-Sopena, Ò. (2016). Interculturalidad y migración: la experiencia educativa y lingüística en el aprendizaje de español en rumanos. Revista Nebrija de $\begin{array}{llll}19 & 9\end{array} \quad$ Lingüística Aplicada (2016) 21.

Van Dijk, T. (1978). La ciencia del texto. Paidós Ibérica. Barcelona.

Vilà, R. (2003). La competencia comunicativa intercultural en educación secundaria obligatoria: Escala de Sensibilidad Intercultural. En E. Soriano-Ayala (Coord.), Pers-pectivas teórico-prácticas en educación intercultural (pp. 103-112). Almería: Servicio de Publicaciones, Universidad de Almería. 